\title{
Ein Parallelkorpus im Einsatz: grammatische Variation im Bereich der Satzverbindung und Informationsverteilung (Deutsch - Norwegisch - Englisch/Französisch) ${ }^{1}$
}

\begin{abstract}
Am Beispiel des an der Universität Oslo entwickelten Oslo Multilingual Corpus (OMC) wird illustriert, wie ein Parallelkorpus aus Originaltexten und deren Übersetzungen zur sprachvergleichenden Erforschung von Phänomenen der Satzverbindung und der Informationsverteilung auf Satz- und Textebene eingesetzt werden kann. Nach einer Skizze der OMC-Architektur wird eine Untersuchung von Satzverknüpfungen mit dem komitativen Konnektor wobei und deren Entsprechungen in norwegischen Übersetzungen und Originaltexten vorgestellt, die dazu beiträgt, Bedeutungsfacetten dieses Konnektors aufzuzeigen, die in rein intralingualen Studien nicht so einfach zu erkennen sind, und dadurch einen besseren und systematischeren Einblick in die angewandten Übersetzungsstrategien gibt. Als zweites Einsatzbeispiel wird eine explorative Untersuchung zur Elaborierung von Ereignisbeschreibungen vorgestellt, die deutsche, norwegische, englische und französische Entsprechungen von mit-Konstruktionen (sog. „Sätzchen“) als Ausgangspunkt nimmt. Beide Studien illustrieren, dass ein Parallelkorpus auch ohne komplexe Annotierungen nicht nur für wort-basierte quantitative Untersuchungen verwertet werden, sondern auch im Zuge weniger zielgerichteter, eher qualitativ angelegter Studien als „Augenöffner“ für komplexe linguistische Phänomene dienen kann.
\end{abstract}

\section{Einleitung}

In diesem Beitrag wird am Beispiel des an der Universität Oslo entwickelten „Oslo Multilingual Corpus“ (OMC) illustriert, wie ein Parallelkorpus aus Originaltexten und deren Übersetzungen für verschiedene Typen sprachkontrastiver bzw. übersetzungswissenschaftlicher Untersuchungen eingesetzt werden kann. Zunächst werden die Architektur des OMC und seine Suchmöglichkeiten vorgestellt und die Typen von Studien skizziert, für die sich diese Art von Korpus primär eignet bzw. die bereits durchgeführt wurden (Abschnitt 2). Anschließend werden zwei eher explorative Einsatzbeispiele vorgestellt, die sich von klassischen quantitativen Korpusstudien, die ihren Ausgangspunkt in der systematischen Untersuchung vorab definierter Para-

Wir danken zwei anonymen Gutachtern für nützliche Kommentare zur ersten Fassung dieses Aufsatzes. 
meter nehmen, unterscheiden, angesichts des im Vergleich zu monolingualen Korpora typischerweise begrenzteren Korpusumfangs aber durchaus typisch für die Verwendung von Parallelkorpora sind: Eine Korpusstudie zu Satzverknüpfungen mit dem W-Präpositionaladverb wobei und deren Entsprechungen in norwegischen Übersetzungen und Originaltexten zeigt Bedeutungsfacetten von Satzverbindungen auf, die in rein mono- bzw. intralingualen Studien nicht so einfach zu erkennen sind (Abschnitt 3). In Abschnitt 4 wird eine OMC-gestützte Untersuchung satzähnlicher Adjunkte (u.a. so genannter small clauses - ,Sätzchen') im Deutschen, Norwegischen, Englischen und Französischen vorgestellt. Abschnitt 5 fasst noch einmal den möglichen Beitrag von Parallelkorpora als „Augenöffner“ für komplexe linguistische Phänomene zusammen.

\section{Das Oslo Multilingual Corpus (OMC)}

Das Oslo Multilingual Corpus ist eine Erweiterung und Weiterentwicklung des English-Norwegian Parallel Corpus (ENPC), eines Parallelkorpus aus fiktionalen und nicht-fiktionalen englischen und norwegischen Originaltexten und deren Übersetzungen in die jeweils andere Sprache, das in den neunziger Jahren an der Universität Oslo im Rahmen eines Forschungsprojektes unter der Leitung von Stig Johansson aufgebaut wurde (für einen Überblick vgl. Johansson 1998 und Oksefjell 1999). Im Rahmen des Projektes SPRIK - Sprache (n) im Kontrast ${ }^{2}$ - wurde das ENPC vor allem um deutsche und französische Texte erweitert und somit zum Oslo Multilingual Corpus (vgl. Tabelle 1). Der Schwerpunkt der Erweiterung lag dabei auf fiktionalen Texten. Der Zugang zum Korpus ist aus urheberrechtlichen Gründen auf Forscher und Studierende der Universitäten Oslo und Bergen beschränkt. Mit Hilfe eines an der Universität Bergen entwickelten Programms, dem „Translation Corpus Aligner" (Hofland/Johansson 1998), sind Originaltexte und Übersetzungen in einem halbautomatischen Verfahren satzweise aneinander ausgerichtet (aligniert).

Um den Umfang der Korpustexte vergleichbar zu halten, sind normalerweise jeweils etwa 10.000 bis 15.000 Wörter vom Anfang eines Textes in das Korpus integriert. Auch bezüglich der Anzahl der Texte pro Übersetzungsrichtung sind die drei Teilkorpora balanciert, was für vergleichende Untersuchungen der beiden Übersetzungsrichtungen entscheidend ist. Tabelle 1 gibt eine Übersicht über die Korpustexte in den einzelnen Teilkorpora. ${ }^{3}$ Die Teilkorpora sind einzeln recherchierbar.

Vgl. www.hf.uio.no/ilos/forskning/prosjekter/sprik/.

Vgl. www.hf.uio.no/ilos/english/services/omc/sub-corpora/. 


\begin{tabular}{|l|l|l|}
\hline Teilkorpus & Originaltexte & Textsorten \\
\hline $\begin{array}{l}\text { ENPC } \\
\text { (Englisch-Norwegisch) }\end{array}$ & $\begin{array}{l}30 \text { englische } \\
30 \text { norwegische }\end{array}$ & fiktional \\
\hline ENPC & $\begin{array}{l}20 \text { englische } \\
20 \text { norwegische }\end{array}$ & nicht-fiktional \\
\hline $\begin{array}{l}\text { GNPC } \\
\text { (Deutsch-Norwegisch) }\end{array}$ & 18 norwegische & fiktional \\
\hline GNPC & 6 norwegische & nicht-fiktional \\
\hline FNPC & 6 deutsche & französische \\
(Französisch-Norwegisch) & 5 norwegische & fiktional \\
\hline FNPC & $\begin{array}{l}10 \text { französische } \\
10 \text { norwegische }\end{array}$ & nicht-fiktional \\
\hline
\end{tabular}

Tab. 1: OMC-Teilkorpora

\begin{tabular}{|l|l|l|}
\hline Teilkorpus & Originaltexte & Textsorten \\
\hline En-De-En & $\begin{array}{l}33 \text { englische } \\
21 \text { deutsche }\end{array}$ & $\begin{array}{l}\text { gemischt } \\
\text { fiktional/nicht-fiktional }\end{array}$ \\
\hline De-No-De & $\begin{array}{l}37 \text { deutsche } \\
38 \text { norwegische }\end{array}$ & $\begin{array}{l}\text { gemischt } \\
\text { fiktional/nicht-fiktional }\end{array}$ \\
\hline No-En-De & 22 norwegische & gemischt fiktional/nicht-fiktional \\
\hline En-De-No & 33 englische & gemischt fiktional/nicht-fiktional \\
\hline De-En-No & 21 deutsche & gemischt fiktional/nicht-fiktional \\
\hline No-Fr-De & 7 norwegische & fiktional (80\% der Texte) \\
\hline No-En-Fr-De & 5 norwegische & fiktional (80\% der Texte) \\
\hline weitere: & En-Niederländisch, En-No-Portug., En-Finn., Russ.-No ${ }^{4}$ \\
\hline
\end{tabular}

Tab. 2: Weitere OMC-Teilkorpora

Es gibt weitere Teilkorpora unterschiedlicher Größe aus Texten mit verschiedenen Sprachkombinationen, teils als Übersetzungskorpora mit Texten in einer Sprache und deren Übersetzung in zwei oder drei andere, teils als Parallelkorpora mit Originaltexten für zwei Sprachen und deren Übersetzung in die jeweils andere (vgl. Tabelle 2). Die in den Teildatenbanken

$4 \quad$ Das russisch-norwegische Parallelkorpus (RuN-Korpus) wurde im Rahmen des Projektes RuN mit den Werkzeugen des OMC entwickelt, wird jedoch als separate Korpusressource verwaltet (vgl. www.hf.uio.no/ilos/english/research/projects/run/corpus/). 
erfassten Texte sind z.T. identisch, beispielsweise enthält das gemischte deutsch-norwegisch-deutsche Korpus weitgehend die gleichen Texte wie die fiktionalen und nicht-fiktionalen Teile des GNPC.

Diese Konstellationen aus Originaltexten und Übersetzungen erlauben verschiedene Typen sprachkontrastiver und übersetzungswissenschaftlicher Untersuchungen wie in Abbildung 1 anhand von drei der Sprachen illustriert: Das Vorkommen sprachlicher Phänomene kann zwischen Originaltexten und ihren Übersetzungen in eine oder mehrere andere Sprachen verglichen werden, z.B. in deutschen Originaltexten und ihren norwegischen und englischen Übersetzungen. Phänomene können auch in Originaltexten im Vergleich zu übersetzten Texten der gleichen Sprache verglichen werden. Eine weitere Möglichkeit ist die Nutzung des OMC als Vergleichskorpus, wobei nur die Originaltexte oder nur die Übersetzungen verschiedener Sprachen untereinander verglichen werden. In diesem Beitrag geht es vor allem um die Untersuchung von Originaltexten und deren Übersetzungen.

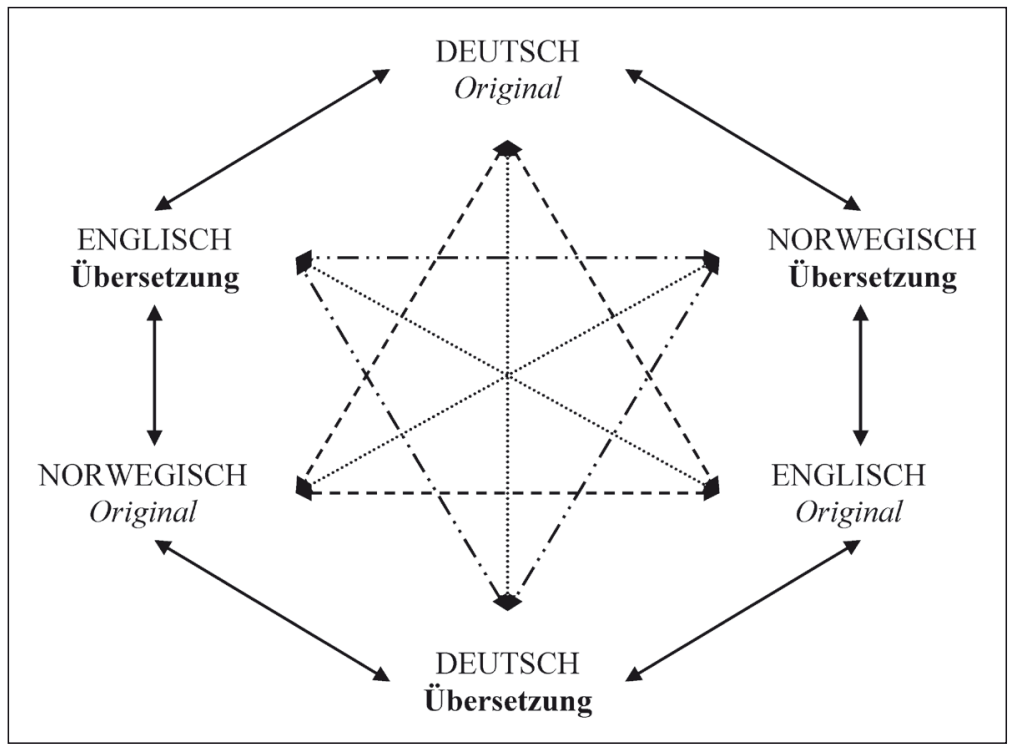

Abb. 1: Dreisprachiges Parallelkorpusmodell

Wie in allen Korpora kann auch im OMC nur nach Informationen gesucht werden, die in irgendeiner Form bereits vorhanden sind, sei es als lexikalische Einheiten in den original- oder zielsprachlichen Texten oder als grammatische Annotationen. Das OMC existiert derzeit in zwei Versionen, die sich in Bezug auf die Verfügbarkeit von Annotationen (Part-of-Speech-Tagging) unterscheiden und die mit zwei verschiedenen Suchwerkzeugen recherchierbar sind. Die ursprüngliche Version ist mit dem Perl-basierten „Translation Corpus Explorer“ (TCE) recherchierbar (Ebeling 1998). Hier sind lediglich 
die Originaltexte des ENPC (also englische und norwegische) nach Wortklassenzugehörigkeit annotiert, Texte in den übrigen Sprachen jedoch nicht. Der TCE erlaubt die Suche nach Wortformen oder Teilen von Wortformen in den Originaltexten oder Übersetzungen, wobei auch die Position relativ zum Satzanfang oder zum Satzende angegeben werden kann. Über verschiedene Filterfunktionen kann weiterhin spezifiziert werden, welche Wortformen oder Teile von Wortformen zusätzlich in Originaltext und/oder Übersetzung vorkommen oder ausgeschlossen werden sollen. Dabei kann auch deren Position relativ zum Suchstring angegeben werden.

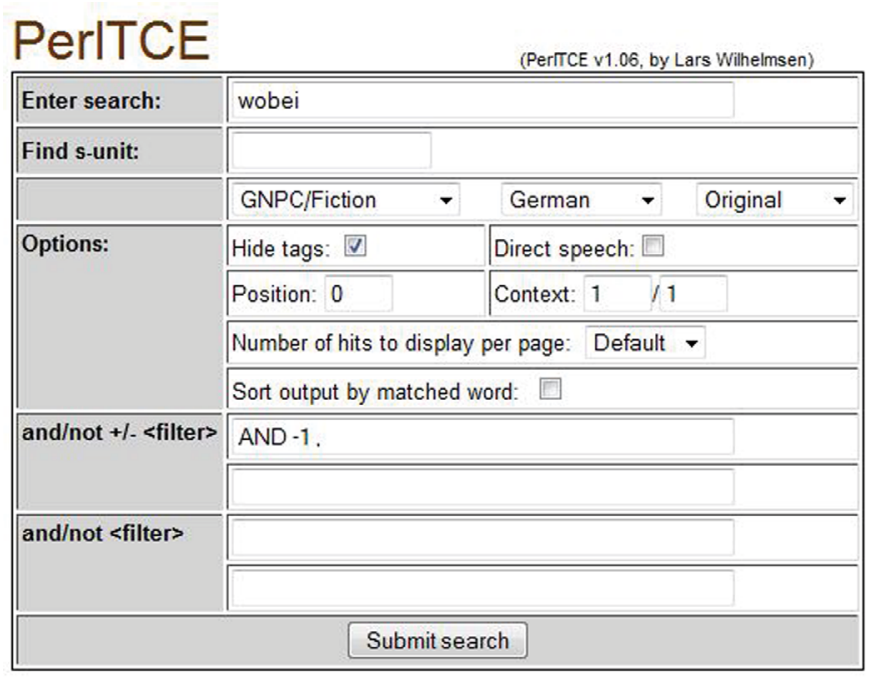

Abb. 2: TCE-Suchmaske

Abbildung 2 zeigt ein Beispiel für die Suche nach dem Vorkommen von wobei in den deutschen Originaltexten des fiktionalen Teils des GNPC. Der erste Filter spezifiziert, dass nur Beispiele mit wobei nach Komma angezeigt werden sollen (keine Beispiele am Satzanfang). Als Kontext sollen jeweils ein Satz vor und nach den wobei-Sätzen und ihren norwegischen Entsprechungen angezeigt werden. Abbildung 3 gibt einen Ausschnitt aus dem Ergebnis der Suchanfrage wieder, das ggf. weiter gefiltert werden kann.

In einer zweiten Version des OMC sind alle Korpustexte nach Wortklassenzugehörigkeit (PoS) annotiert und es stehen mit Hilfe der Schnittstelle „Glossa“" (Nygaard et al. 2008) avanciertere Suchmöglichkeiten zur Verfügung. „Glossa“" wurde am Textlaboratorium der Universität Oslo entwickelt und wird auch für andere, vor allem einsprachige norwegische Korpora verwendet. ${ }^{5}$ Diese OMC-Version ist jedoch noch in der Testphase, weswegen sie erst eingeschränkt für kontrastive Studien genutzt wird.

Vgl. www.hf.uio.no/iln/english/about/organization/text-laboratory/services/glossa.html. 


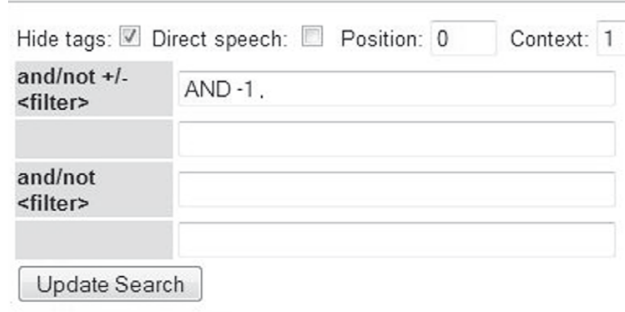

Total before filters: 19 .

wobei : 16

Results: 1 to 16 (after filters)

Ich glaubte, die Blicke der Frau in meinem Rücken zu spüren.

Nach einer Wegbiegung nahm ich endlich den Mantel ab und legte ihn über den Arm, wobei ich mich umdrehte.

Ich sah nur Gräber und unbewegliche, staubige Bäume.

(CH1)

Jeg syntes at jeg merket blikket fra kvinnen mot ryggen min

Etter en sving tok jeg endelig av meg kåpen, la den over armen og snudde meg.

Jeg så bare graven og ubevegelige, støvete træer.

(CH1TN)

Apollon, der Gott der Seher.

Der wußte, was ich heiß begehrte: die Sehergabe, die er mir durch eine eigentlich beiläufige, ich wagte nicht zu fühlen: enttäuschende Geste verlieh, nur um sich mir dann als Mann zu nähern, wobei er sich - ich glaubte, allein durch meinen grauenvollen Schrecken - in einen Wolf verwandelte, der von Mäusen umgeben war und der mir wütend in den Mund spuckte, als er mich nicht überwältigen konnte.

So daß ich beim entsetzten Erwachen einen unsagbar widerwärtigen Geschmack auf der Zunge spürte und mitten in der Nacht aus dem Tempelbezirk, in dem zu schlafen ich zu jener Zeit verpflichtet war, in die Zitadelle, in den Palast, ins Zimmer, ins Bett der Mutter floh.

(CW1)

Apollon, seernes gud.

Han kjente mitt hete begjæer etter seergaven, som han gav meg næermest i forbifarten, jeg våget ikke å kjenne etter, med en tilfeldig, skuffende håndbevegelse, bare for så å nærme seg meg som mann. Dertil forvandlet han seg — på grunn av min ubeskrivelige redsel, tror jeg — til en ulv, som var omgitt av mus, og som spyttet meg rasende i munnen da han ikke kunne få bukt med meg.

Så da jeg våknet, kjente jeg en ubeskrivelig ekkel smak på tungen og flyktet midt på natten ut fra tempelområdet, hvor jeg pá denne tiden var forpliktet til â sove, inn i citadellet, inn i palasset, inn på rommet til mor og ned $\mathrm{i}$ sengen hennes.

(CW1TN)

Abb. 3: Ergebnis der TCE-Suchanfrage

Grundsätzlich eignen sich jedoch beide Schnittstellen besonders für kontrastive Untersuchungen, die an konkreten lexikalischen Einheiten wie z.B. Konnektoren, Verben oder Präpositionen festgemacht werden können oder diese als Ausgangspunkt nehmen. Trotz dieser Beschränkungen ist mit dem OMC bereits ein breites Spektrum von Phänomenen für die verschiedenen Korpussprachen kontrastiv untersucht worden (Johansson 2007): Beispiele für das Sprachpaar Norwegisch-Englisch sind Untersuchungen zu Spaltsatzkonstruktionen (Ebeling 2000), zur Besetzung der Thema- bzw. Topikposition (Hasselgård 2007) oder zur Modalität mit Ausgangspunkt in der Verwendung von Modalverben (Løken 2007). Für das Deutsche und Norwegische wurden beispielswese Adverbialsätze im Grenzbereich komitativ-instrumen- 
tal untersucht (Giesebrecht 1999), ein anderes Beispiel sind repetitive bzw. restitutive Konstruktionen mit wieder im Deutschen vs. igjen im Norwegischen (und again im Englischen) (Fabricius-Hansen 2004, 2005).

Im Folgenden werden zwei Beispiele für die Nutzung des OMC präsentiert, die im Rahmen eines Buchprojektes der SPRIK-Gruppe mit dem Titel Big Events, Small Clauses: the Grammar of Elaboration (Fabricius-Hansen/Haug (Hg.) i.Dr.) entstanden sind: eine zielgerichtete korpusbasierte Untersuchung zum deutschen komitativen Konnektor wobei und dessen Übersetzung ins bzw. aus dem Norwegischen (Abschnitt 3) und eine explorative Studie, die sich, von der prototypischen komitativen Präposition mit (bzw. med, with, ...) ausgehend, mit satzähnlichen, aber nicht finiten Mitteln zur Elaborierung von Ereignisbeschreibungen befasst (Abschnitt 4), wie sie in (1) und (2) veranschaulicht werden. Dieses Thema wird in größerer Ausführlichkeit in dem Buchprojekt behandelt.

(1) Dann kommt Kusna ganz, empört zurück, einen Brief in der Hand.

(2) With a glass of wine in his hand, he stood smiling in front of the house, leaning slightly backward, his legs wide apart.

Der Themenbereich wurde unter verschiedenen Perspektiven und für verschiedene Sprachen bzw. Sprachkombinationen untersucht.

\section{Wobei-Sätze und ihre norwegischen Entsprechungen in Übersetzungen und Originaltexten}

\subsection{Kontext der Untersuchung}

Den Kontext der Korpusrecherche von Satzverknüpfungen mit dem komitativen W-Präpositionaladverb wobei bildete eine Untersuchung zu den Diskurseigenschaften von nicht-restriktiven Relativsätzen im Deutschen und deren norwegischen Übersetzungen (Ramm 2011), im Falle der wobei-Sätze auch von deren norwegischen Entsprechungen in Übersetzungen aus dem Norwegischen (Ramm i.Dr.). Sowohl bei nomenbezogenen als auch bei satzbezogenen Relativsätzen gibt es Unterschiede im Sprachsystem der beiden Sprachen, die dazu führen, dass in der Übersetzung häufig keine entsprechende Relativsatzkonstruktion gewählt werden kann, sondern in verschiedener Weise umstrukturiert werden muss. Satzbezogene, mit W-Präpositionaladverbien wie womit, weshall, wodurch etc. oder mit was eingeleitete Relativsätze sind im Norwegischen weniger gebräuchlich bzw. wirken teilweise veraltet. Lediglich für den Relativmarker was gibt es ein unmarkiertes norwegisches Äquivalent, noe (som), das auch tatsächlich häufig als Entsprechung in der norwegischen Version zu finden ist. Ansonsten ist es eine gän- 
gige Übersetzungsstrategie, dass der Relativsatz zum Hauptsatz hochgestuft wird, wobei teilweise auch ein Punkt gesetzt wird, also eine neue Satzgrenze eingeführt wird. Die Beziehung zwischen den Sätzen kann dabei mit alternativen Konnektoren signalisiert sein, beispielsweise mit einem korrespondierenden Präpositionaladverb wie derfor (,deshalb) als Entsprechung von weshalb, sie kann aber auch implizit bleiben (Ramm 2011).

Satzbezogene Relativsätze gehören zu den so genannten weiterführenden Relativsätzen: Obwohl sie syntaktisch dem Matrixsatz subordiniert sind, erkennbar beispielsweise an der Endstellung des finiten Verbs, haben sie ansonsten viel Ähnlichkeit mit unabhängigen Hauptsätzen, wie Holler (2005) in ihrer Arbeit zu nicht-restriktiven Relativsätzen im Deutschen aufzeigt (zu weiterführenden Nebensätzen im Deutschen vgl. auch Brandt 1990 und Peyer 1997). Trotz ihrer Hauptsatzähnlichkeit sind sie jedoch nicht ganz mit diesen äquivalent, was wiederum im Übersetzungszusammenhang interessant ist, da die syntaktische Hochstufung zum selbstständigen Hauptsatz (in Kombination mit anderen Umstrukturierungen) wie erwähnt eine typische Übersetzungslösung sein kann. Es wurde daher untersucht, welche Konsequenzen solche z.T. unvermeidlichen Umstrukturierungen für die Beziehungen zwischen den Sätzen haben, d.h. ob sich Originaltext und Übersetzung dadurch in ihren Diskurseigenschaften unterscheiden (Ramm 2011).

Relativsätze mit wobei sind aus Übersetzungsperspektive besonders interessant, weil es im Norwegischen (wie in vielen anderen Sprachen) weder für das W-Präpositionaladverb wobei noch für das korrespondierende Präpositionaladverb dabei eine Entsprechung gibt. Die Bedeutung der Satzverknüpfung muss entweder durch andere Mittel wiedergegeben oder implizit gelassen werden. Wobei hat eine transparente lexikalische Struktur, bestehend aus dem referenziell-deiktischen Element wo und der semantisch ausgesprochen unterspezifizierten Präposition bei (Fabricius-Hansen 1999; Grabski/Stede 2006). Eine Frage, die sich für eine kontrastive Untersuchung stellt, ist, ob die beiden lexikalischen Bestandteile von wobei eine Entsprechung in der norwegischen Version haben, auch wenn es kein entsprechendes W-Präpositional- oder Präpositionaladverb gibt. Eine andere Frage ist, welche Rolle die komplexe Semantik von Satzverknüpfungen mit wobei für die Art der norwegischen Entsprechung spielt. Wobei ist einer der komitativen Konnektoren, zu denen auch dabei und indem gehören. Ihre Kernbedeutung besteht darin, zwei kookkurrierende Ereignisse in ein Haupt- und ein Nebenereignis zu strukturieren (Breindl in Vorbereitung). Letztendlich entscheidet aber der Typ der verknüpften Prädikate über die Bedeutung, und gerade bei wobei und dabei gibt es häufig auch assoziative Verknüpfungen von Nicht-Ereignisprädikaten, bei denen die temporalen Eigenschaften weniger relevant sind als bei der Verbindung von Ereignisprädikaten (ebd.). 


\subsection{Korpusstudie}

Vor diesem Hintergrund wurde untersucht, welche Entsprechungen wobeiSätze in norwegischen Übersetzungen und Originaltexten haben und mit welchen Typen von wobei-Verknüpfungen sie korrelieren. Als Korpora dienten die fiktionalen und nicht-fiktionalen Teilkorpora des OMC sowie ein weiteres Teilkorpus aus nicht-fiktionalen Texten. ${ }^{6}$ Insgesamt wurden 38 wobei-Sätze in deutschen Originaltexten und 31 in deutschen Übersetzungen untersucht.

Die Untersuchung ergab, dass sich wobei-Verknüpfungen in fiktionalen und nicht-fiktionalen Texten deutlich unterscheiden und dass dies Konsequenzen dafür hat, wie sie übersetzt sind bzw. welche Entsprechungen im norwegischen Ausgangstext zu finden sind, also welche Bedeutungsfacette der Satzverknüpfung in der norwegischen Version wiederzufinden ist. So werden in fiktionalen Texten mit vielen narrativen Textpassagen in wobeiSätzen eher Ereignisprädikate verknüpft bzw. ein Ereignisprädikat mit einem Zustandsprädikat. Dadurch steht eher der kotemporale Aspekt der Satzverknüpfung im Vordergrund. In der norwegischen Version findet man hier typischerweise temporale Adverbialsätze oder aber Koordinationen mit $g g$ (,und).

In Beispiel (3) $)^{7}$ ist ein wobei-Satz als Adverbialsatz mit mens (,während) wiedergegeben. Die Versionen sind zwar sehr ähnlich, doch gibt die Übersetzung nur den temporalen Aspekt der Verknüpfung wieder, während der wobeiSatz neben der Kotemporalität auch etwas über die Gewichtung der verknüpften Ereignisse in ein Haupt- und ein Nebenereignis ausdrückt oder zumindest das Potenzial dazu hat. In (4) bildet ein Adverbialsatz mit dem etymologisch mit dem deutschen indem verwandten idet die Ausgangsstruktur für eine Übersetzung als wobei-Satz. Indem hat jedoch eine deutlich instrumentalere Bedeutung als idet (Giesebrecht 1999), weshalb eine parallele Übersetzung mit indem hier nicht in Frage kommt.

(3) D Ohne ein Geräusch zu machen ging er rückwärts auf die Ladentür zu, wobei er die andere Tür, die zum Kabinett, ängstlich im Auge behielt. (ME1)

$\mathrm{N}$ Uten en lyd listet han seg baklengs mot butikkdøra, mens han hele tida engstelig holdt øye med den andre døra, den til bakværelset. (ME1TN)

Obne ein Geräusch schlich er sich rückwärts zur Ladentür, während er die ganze Zeit ängstlich im Auge bebielt die andere Tür, die zum Hinterzimmer.

Das Teilkorpus wurde mit den OMC-Werkzeugen im Zusammenhang mit der Untersuchung von Ramm (2011) erstellt, ist aber noch nicht in das OMC integriert.

Im Anschluss an die norwegische Version ist bei diesem und den folgenden Beispielen eine quasi-wörtliche deutsche Entsprechung (Glossierung) angegeben. 
(4) N „Egentlig Per Kristian,“ sier han på samme tid brydd og overbærende $i d e t$ han reiser seg fra en lav, bred sofabenk full av puter $\mathrm{i}$ alle farger. (KF1)

„Eigentlich Per Kristian,“ sagt er gleichzeitig gequält und nachsichtig indem er sich erhebt von einer niedrigen, breiten Sofabank voll mit Kissen in allen Farben.

D „Eigentlich Per Kristian“ sagt er im gleichen Augenblick, verlegen und nachsichtig, wobei er sich von einem niedrigen breiten Sofa, das mit Kissen in allen Farben übersät ist, erhebt. (KF1TN)

Als norwegische Entsprechungen von wobei-Sätzen findet man auch Satzoder VP-Koordinationen mit og (,und') wie in (5). Auch hier sieht man, dass sich durch die unvermeidliche Wahl einer alternativen Verknüpfung die Bedeutung in der Übersetzung leicht verschiebt: Während in der deutschen Version der wobei-Satz durch die syntaktische Subordination und die Semantik von wobei eher als kotemporales Nebenereignis gekennzeichnet ist, liest man die Norwegische als narrative Folge von drei Ereignissen (den Mantel abnehmen, ihn über den Arm legen, sich umdrehen). Die Verwendung von wobei in der deutschen Version blockiert jedoch eine sequenzielle Lesart (des dritten Ereignisses) und erzwingt eine kotemporale Interpretation (vgl. FabriciusHansen 2005 für einen entsprechenden Effekt von dabei).

D Nach einer Wegbiegung nahm ich endlich den Mantel ab und legte ihn über den Arm, wobei ich mich umdrehte. (CH1)

$\mathrm{N}$ Etter en sving tok jeg endelig av meg kåpen, la den over armen og snudde meg. (CH1TN)

Nach einer Kurve nabm ich endlich den Mantel ab, legte ihn über den Arm und drebte mich um.

Die nicht-fiktionalen Korpustexte enthalten neben kotemporalen wobei-Beispielen auch viele assoziative Verwendungen von wobei, bei denen Nicht-Ereignisprädikate verknüpft sind und folglich die temporale Struktur weniger relevant ist. Dieser Unterschied schlägt sich auch bei den Übersetzungsstrategien nieder. In der Übersetzung wird hier eher die referenziell-deiktische Komponente (also eine Entsprechung von wo-) wiedergegeben wie in (6) oder die Beziehung wird implizit gelassen wie in (7):

(6) D Seit der zweiten Hälfte der dreißiger Jahre befasste sich die deutsche Marineführung mit dem „Fall Norwegen“, wobei sowohl defensive, als auch offensive Motive eine Rolle spielten. Durch den Erwerb von Stützpunkten sollte die Basis für eine Seekriegführung im Atlantik gelegt werden, [...]. (BB1)

$\mathrm{N}$ Fra annen halvdel av 30-årene beskjeftiget den tyske marineledelsen seg med „Fall Norwegen“. Både defensive og offensive motiver spilte her en rolle. Erobringen av støttepunkter skulle legge grunnlaget for sjøkrigføringen i Atlanterhavet, [...]. (BB1TN) 
Seit der zweiten Hälfte der 30-er Jahre beschäftigte die deutsche Marineführung sich mit „Fall Norwegen“. Sowohl defensive und offensive Motive spielten hier eine Rolle. Die Eroberung von Stützpunk.ten sollte legen die Grundlage für die Seekriegsführung im Atlantik, [...].

(7) D Neben diesen engen wirtschaftlichen Kontakten bestanden rege kulturelle, technische und wissenschaftliche Beziehungen, wobei für das norwegische Bildungsbürgertum die deutsche Literatur und die deutsche Sprache den Bezugspunkt darstellten. Zahlreiche skandinavische Studenten studierten in Deutschland, und zwischen den protestantischen Kirchen existierten enge Bindungen. (BB1)

N Ved siden av disse tette handelsforbindelsene fantes det også viktige kulturelle, tekniske og vitenskapelige kontakter. Tysk språk og litteratur stod sterkt i de utdannede borgerlige kretser. Utallige skandinaviske studenter studerte $\mathrm{i}$ Tyskland, og det fantes tette bånd mellom de protestantiske kirkene. (BB1TN)

Neben diesen dichten Handelsverbindungen gab es auch wichtige kulturelle, technische und wissenschaftliche Kontakte. Deutsche Sprache und Literatur stand stark in den ausgebildeten bürgerlichen Kreisen. Unzählige skandinavische Studenten studierten in Deutschland, und es gab enge Verbindungen zwischen den protestantischen Kirchen.

Sowohl in (6) als auch in (7) ist der Relativsatz zum selbstständigen Satz hochgestuft. In (6) kann das deiktische Adverb her (,hier) als Gegenstück zur wo-Komponente identifiziert werden. In (7) muss die Beziehung zwischen den Sätzen jedoch aus dem Kontext erschlossen werden. Insbesondere in (7) ist erkennbar, dass die (unvermeidliche) syntaktische Hochstufung des Relativsatzes und das Weglassen des Konnektors textuelle Konsequenzen haben kann: Die norwegische Version wirkt (zumindest aus Sicht des Deutschen) ,zerhackter“, da die Beziehung zwischen den Sätzen weder syntaktisch noch lexikalisch signalisiert ist - der zweite und dritte Satz sind hier am plausibelsten als separate Elaborierungen des ersten Satzes, der Entsprechung des deutschen Matrixsatzes, zu interpretieren. In der deutschen Version interpretiert man das wobei-Gefüge eher als Einheit (der wobei-Satz drückt einen Nebenaspekt zum Matrixsatz aus), die im nachfolgenden Satz weiter erläutert wird. Ansatzweise ist eine solche Veränderung der Textsegmentierung auch in (6) zu erkennen, doch signalisiert hier zumindest das deiktische her (,hier') den Bezug zum vorangehenden Satz.

\subsection{Weitere Ergebnisse}

Durch die Untersuchung der wobei-Entsprechungen in fiktionalen und nichtfiktionalen Texten wurde deutlich, dass und weshalb verschiedene wobei-Verwendungen typischerweise unterschiedliche norwegische Entsprechungen 
haben. Ein Vergleich mit dem Vorkommen anderer W-Präpositionaladverbien in den OMC-Korpora hat ergeben, dass wobei das am häufigsten vorkommende W-Präpositionaladverb ist, was vielleicht mit seiner äußerst flexiblen Bedeutung zu erklären ist. Eine weitere Korpussuche hat gezeigt, dass wobei-Sätze vergleichsweise häufiger in deutschen Originaltexten als in deutschen Übersetzungen vorkommen, also ein typisches Phänomen deutscher Originaltexte sind. Bei den deutschen Übersetzungen hat wobei zudem fast immer einen norwegischen Konnektor wie mens (,während'), idet (,indem') oder og (,und') als Vorlage, implizite Relationen im norwegischen Original sind also selten, während in den norwegischen Übersetzungen von wobei-Sätzen, vor allem bei den nicht-fiktionalen Texten, bei fast einem Viertel der Beispiele die Beziehung zwischen den Sätzen nicht explizit durch einen alternativen Konnektor oder ein deiktisches Element signalisiert wird.

\section{MIT-Sätzchen und die Elaborierung von Ereignisbeschreibungen ${ }^{8}$}

In diesem Abschnitt wenden wir uns dem Thema zu, das den Kern der im Abschnitt 2 erwähnten, im Rahmen des SPRIK-Projekts entstandenen Publikation Big Events, Small Clauses: the Grammar of Elaboration (Fabricius-Hansen/Haug (Hg.) i.Dr.) bildet. Die sprachlichen Erscheinungen, um die es uns dabei geht, sind in (1) und (2), unten als (8) und (9) wiederholt, kursiv hervorgehoben.

(8) Dann kommt Kusna ganz, empört zurück, einen Brief in der Hand.

(9) With a glass of wine in his hand, he stood smiling in front of the house, leaning slightly backward, his legs wide apart.

Es soll hier allerdings kein Ergebnisbericht erstattet, sondern lediglich der Weg zum Thema, und zwar insbesondere die Rolle des Parallelkorpus auf diesem Weg skizziert werden.

\subsection{Am Anfang war das Wort MIT}

Wie aus Abschnitt 2 hervorgeht, sind die aktuellen Teilkorpora des OMC verhältnismäßig klein und eignen sich deswegen am besten für das Studium von (einigermaßen zuverlässig identifizierbaren) Phänomenen, die eine relativ hohe Textfrequenz aufweisen, wie etwa die üblichsten Konnektoren und Präpositionen. Unser Interesse für die oben erwähnten Konstruktionstypen fing nun mit der folgenden Frage an:

Cathrine Fabricius-Hansen widmet Abschnitt 4 dieses Aufsatzes Brigitte Handwerker zum 60. Geburtstag. 
Inwieweit stimmen die etymologisch verwandten komitativen Präpositionen mit und norw. med syntaktisch und semantisch miteinander (und mit „komitativen“ Präpositionen in anderen Sprachen") überein?

Für Fragestellungen dieser Art ist ein Parallelkorpus wie das OMC ein sehr nützliches Werkzeug, da man gezielt nach Präpositionsvorkommen in Originaltexten und Übersetzungen suchen kann, bei denen das jeweilige lexikalische Pendant (nicht) als Übersetzung bzw. Vorlage dient. So lässt sich untersuchen, wie häufig MIT-Phrasen ${ }^{10}$ in Originaltexten und Übersetzungen (nicht) miteinander korrespondieren, und welche anderen Präpositionen unter welchen Bedingungen als Übersetzungsbild (d.h. Übersetzung oder Vorlage) der Präposition vorkommen - ein erster Schritt in Richtung einer übersetzungsbasierten (kontrastiven) Präpositionssemantik. ${ }^{11}$

Was die Häufigkeit von Nicht-Korrespondenzen betrifft, erhalten wir etwa für das deutsch-norwegische Teilkorpus GNPC Fiction des OMC (in der TCE-Suchmaske, siehe Abschnitt 2) die in Zeile 1 von Tabelle 3 dargestellten Ergebnisse (OT: Originaltexte, ÜT: Übersetzungen): Norw. med wird in $1.124(34 \%)$ von 3.354 Originalfällen nicht mit mit wiedergegeben und geht in 1.291 (38\%) von 3.430 Übersetzungsfällen nicht auf mit zurück. ${ }^{12}$ Für mit ist der Anteil von Nicht-Korrespondenzen in diesem Teilkorpus etwas niedriger (jeweils $21 \%$ und $19 \%$, vgl. Tabelle 3, Zeile 2). ${ }^{13}$ Beispiele für rein lexikalische Nicht-Korrespondenz bieten (10) und (11), wo norw. med jeweils mit bei und in übersetzt wird.

\begin{tabular}{|l|ll|lc|}
\hline \multirow{3}{*}{ 1. med } & \multicolumn{2}{|l|}{ OT-Vorkommen: 3.354} & \multicolumn{2}{l|}{ ÜT-Vorkommen: 3.430} \\
\cline { 2 - 5 } & mit in ÜT: & nicht mit in $\ddot{\mathrm{UTT}}:$ & mit in OT: & nicht mit in OT: \\
& $2.230(66 \%)$ & $1.124(34 \%)$ & $2.139(62 \%)$ & $1.291(38 \%)$ \\
\hline \multirow{3}{*}{ 2. mit } & OT-Vorkommen: 2.492 & ÜT-Vorkommen: 2.690 \\
\cline { 2 - 5 } & med in ÜT: & nicht med in ÜT: & med in OT: & nicht med in OT: \\
& $1.960(79 \%)$ & $\underline{532(21 \%)}$ & $2.187(81 \%)$ & $503(19 \%)$ \\
\hline
\end{tabular}

Tab. 3: Nicht-Übereinstimmung zwischen med und mit in GNPC Fiction

9 Siehe Stolz (1997) und Stolz et al. (Hg.) (2006) für eine typologisch-vergleichende Beschreibung komitativer Präpositionen im weiteren Sinne.

10 MTT als Abkürzung für med/mit/with/avec/...

11 Siehe Solstad (2007) für eine OMC-basierte Implementierung der von Dyvik (1998) entwickelten Methodik am Beispiel durch.

12 Nicht-Korrespondenzen für med wurden mit der Bedingung „NOT mit“ (in der TCE-Suchmaske) für die deutschen Paralleltexte (jeweils Übersetzungen und Originaltexte) herausgefiltert; entsprechend wurde bei mit vorgegangen.

13 Ein Caveat ist hier am Platz: Da in den Parallelkorpora nur Sätze, nicht Phrasen oder Wörter aligniert sind, ist den Befunden in Tabelle 1 nicht direkt zu entnehmen, wie viele med-Vorkommen tatsächlich durch mit übersetzt werden bzw. mit übersetzen, oder umgekehrt, sondern nur, in wie vielen Fällen ein Satz, der med enthält, als Übersetzung bzw. Vorlage einen Satz hat, in dem mit vorkommt, und entsprechend für mit. 
(10) N Det skjer iallfall langt oftere enn med et bilde. (JW1)

D Jedenfalls geschieht es viel öfter als bei einem Bild.

(11) N ,Jeg så en hær av kvitøyer med bla jakker da jorden var rødbrun“, sa Cochise. (SH1)

D „Ich habe ein Heer von Bleichgesichtern in blauen Jacken gesehen, als die Erde rotbraun war", sagte Cochise.

Eine zweite Variante der Nicht-Übereinstimmung liegt vor, wenn die MITPhrase und ihre Übersetzung/Vorlage schon auf der syntaktischen Ebene voneinander abweichen. Eine interessante Frage ist dabei, inwieweit es wiederkehrende oder typische Muster der syntaktisch nicht-analogen Übersetzungsbilder gibt. Und damit sind wir bei unserem Haupthema angelangt.

\subsection{Ein Sonderfall: MIT-Sätzchen}

Bei näherem Hinsehen stellt sich heraus, dass syntaktische Nicht-Korrespondenz nicht selten eine spezifische MIT-Konstruktion involviert, die wir im Anschluss an Gadourek (2006) als MIT-Sätzchen bezeichnen, und zwar oft dergestalt, dass das MIT-Sätzchen im Norwegischen (Original oder Übersetzung) erscheint, aber nicht im Deutschen oder Englischen (oder Französischen), wo sich stattdessen oft eine so genannte Absolutkonstruktion findet; vgl. (12) bis (14). ${ }^{14}$

(12) N Til slutt var det ingen annen utvei enn å løpe fra ham som en annen tyv, med fiolinkassen og kofferten under armene. (EFH1) ..., mit geigenkasten.DEF und koffer.DEF unter arm.PL.DEF

D Zuletzt gab es keinen anderen Ausweg, als vor ihm wegzurennen wie ein Dieb, unter den Armen Geigenkasten und Koffer.

E Finally David had no way out except to run away like any thief, his violin and suitcase under his arms.

(13) N De ble stående sie blieben stehend

med celloen mellom seg. (HW2) mit cello.DEF zwischen REFL.

D Sie standen einander gegenüber, das Cello zwischen sich.

F Ils étaient debout, le violoncelle entre eux. ..., das Cello zwischen PRON

(14) D Seff jedoch, sein Vater, saß eingesunken in der Kirchenbank, den Blick tief in die Knie gebohrt. (ROS1)

N Men Seff, hans far, satt sammensunket på kirkebenken, $\underline{\text { med blik- }}$ ket boret dypt ned $i$ knarne. ..., mit blick.DEF gebohrt tief runter in knie.PL.DEF

14 In den Beispielblöcken aus dem OMC steht der Beleg aus dem Originaltext immer zuerst, mit einem Kürzel für den betreffenden Text. 
Die Absolutkonstruktionen sind als, small clauses“ (SCs), Sätzchen, zu analysieren: Sie bestehen aus einer Nominalphrase (DP) und einer nicht-finiten XP - Präpositionalphrasen in (12) und (13), Partizipialphrase in (14) -, zwischen denen semantisch gesehen eine Prädikationsrelation besteht, wobei XP als Prädikat und die DP, die im Deutschen den Akkusativ aufweist, als Argument dient (Bech 1955, S. 56 ff.; Zifonun/Hoffmann/Strecker 1997, S. 2224 2226); vgl. (15).Wir werden die DP im Folgenden als das SC[-interne]-Subjekt bezeichnen. ${ }^{15}$

$$
\begin{gathered}
\text { [sc [DP den Blick] } \\
\text { SC-Subjekt } \\
\text { [partP tief in die Knie gebohrt]] } \\
\text { SC-Prädikat }
\end{gathered}
$$

Das Deutsche kennt neben dem in (14) veranschaulichten absoluten Akkusativ eine (scheinbar) ähnliche Konstruktion mit dem internen Subjekt im Nominativ (Zifonun/Hoffmann/Strecker 1997, S. 2225; Blühdorn in diesem Band, Abschnitt 4.1); vgl. (16 D) und Beispiel (19) in Blühdorn (ebd.).

(16) N Nå er det jeg som er på eventyr, stadig mer overmodig med kroppen $i$ spenn. (SL1)

jetzt ist es ich REL ist auf abenteuer, immer mehr übermütig mit körper.DEF in Spannung

D Jetzt bin nämlich ich auf Abenteuer aus, immer übermütiger, mein Körper gespannt wie ein Fliť̨bogen.

Diese Konstruktion unterscheidet sich jedoch in einem zentralen Punkt vom absoluten Akkusativ (Gadourek 2006): Reflexiva im Prädikatsteil der absoluten Akkusativkonstruktion beziehen sich notgedrungen auf eine DP im Matrixsatz, und zwar meistens auf dessen Subjekt, wie sich in (17a) (vgl. Bech 1955; Vuillaume 1995); d.h. sie werden vom Matrixsatz aus gebunden. Entspechendes ist in der Nominativkonstruktion nicht möglich; der Binder muss hier das interne nominativische Subjekt sein. (17b) kann somit nicht im Sinne von (17a) interpretiert werden und ist in dem strukturell lizensierten Sinne semantisch-pragmatisch abweichend. Aus diesem Grunde sind absolute Nominative grundsätzlich anders zu analysieren als absolute Akkusative, und zwar als ,echte' Sätze mit elliptischem finitem Verb (Gadourek 2006).

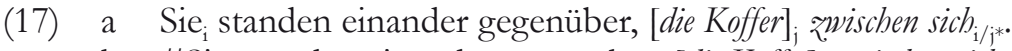

b \#Sie $\mathrm{i}_{\mathrm{i}}$ standen einander gegenüber, [die Koffer $]_{\mathrm{j}}$ zwischen sich $_{\mathrm{i}^{*} / \mathrm{j}}$

Da jedoch der Kasus des SC-Subjekts außer im Maskulinum Singular morphologisch unterspezifiziert (Akkusativ/Nominativ) ist und der Prädikats-

15 In der älteren Fachliteratur wird der absolute Akkusativ, oft eine Partizipialphrase mit elliptischem habend oder haltend als Kopf analysiert. Wie Zifonun/Hoffmann/Strecker (1997, S. 2224) zeigen, ist diese Analyse jedoch nicht haltbar; vgl. z.B. *den Blick in die Knie gebohrt habend. 
teil eher selten ein eindeutiges Reflexivum enthält, lassen sich Absolutkonstruktionen im Deutschen oft nicht eindeutig als absolute Akkusative bzw. Nominative bestimmen. Auch für das Norwegische, das wie das Deutsche echte Reflexiva besitzt, sind aufgrund der Bindungsverhältnisse zwei verschiedene Konstruktionstypen anzusetzen. Wir können diese Thematik jedoch hier nicht weiter verfolgen.

Interessanterweise muss nun für die MIT-Sätzchen, abgesehen von MIT selber, dieselbe zweigliedrige SC-Struktur angesetzt werden wie für ,echte (d.h. nicht-nominativische) Absolutkonstruktionen; die Bindungsregularitäten sind, wie (18) zeigt, auch die gleichen.

(18) $\mathrm{Sie}_{\mathrm{i}}$ standen einander gegenüber, [?pp mit ${ }_{\mathrm{SC}}\left[{ }_{\mathrm{DP}} \text { den Koffern }\right]_{\mathrm{j}}\left[_{\mathrm{pp}}\right.$ zwischen $\left.\left.\left.\operatorname{sich}_{\mathrm{i} / * j}\right]\right]\right]$

MIT zeigt in diesen Fällen mit anderen Worten als „Präpositionalsubjunktion“ (Gadourek 2006) eine Art Zwitternatur: Das Wort regiert wie eine normale Präposition den morphologischen Kasus der nachfolgenden DP (Dativ im Deutschen), benimmt sich jedoch wie eine Subjunktion, insofern es eine satzähnliche Phrase als Komplement nimmt.

Während eine SC-Analyse der einschlägigen Konstruktionen für das Englische geläufig ist (Jespersen 1940; McCawley 1983; Kortmann 1995 u.a.) und auch für das Französische (Ruwet 1978; Chuquet/Chuquet 2006) und Norwegische (Aa 2006) vorgeschlagen worden ist, hat der Konstruktionstyp im Deutschen wenig Beachtung gefunden (siehe Gadourek 2006). Zifonun/Hoffmann/Strecker (1997, S. 2139) veranschaulichen ihn zwar anhand von (19) und charakterisieren ihn als ,verwandt mit“ dem im konkreten Beispiel parataktisch angeschlossenen absoluten Akkusativ (den offenen Hut neben mir), scheinen ihn aber im Hinblick auf die interne Struktur der mit-Phrase nicht als einen Sonderfall zu beachten; mit wird vielmehr als eine normale Präposition und in der Hand als Attribut zu (der) Gitarre aufgefasst, wie in (20) veranschaulicht; Ähnliches trifft auf Stolz et al. (Hg.) (2006) zu.

Mit der Gitarre in der Hand, den offenen Hut neben mir, mit meinem wahren Gesicht würde ich auf den Zug aus Rom warten. (Zifonun et al. 1997, S. 2139)

$$
\begin{aligned}
& \text { a } \left.\quad\left[{ }_{\mathrm{pp}} \underline{\text { Mit }}{ }_{{ }_{\mathrm{DP}}} \operatorname{der}\left[{ }_{\mathrm{NP}}[\mathrm{Np} \text { Gitarre }]{ }_{\mathrm{pp}} \text { in der Hand] }\right]\right]\right] \ldots \\
& \text { b } \quad\left[\mathrm { pp } \text { Mit } \left[_{\mathrm{DP}}[\mathrm{DP} \text { der Gitarre] [pp in der Hand] ] ... }\right.\right.
\end{aligned}
$$

Auf den ersten Blick leuchtet die Unangemessenheit der in (20) veranschaulichten MIT-DP-Analyse vielleicht nicht ein. Sie beinhaltet jedoch, dass das MIT-Komplement als (komplexe) DP auch allein auftreten kann, und das dürfte in der Tat eher die Ausnahme als die Regel sein, wie Gadourek (2006, S. 46 f.) bemerkt. So scheidet die DP-Analyse aus, wenn das Sätzchen eine 
Partizipialphrase als zweiten (Prädikats-)Teil enthält, wie meistens auch bei direktiven Präpositionalphrasen und Präpositionaladverbien als Prädikatsteilen; vgl. (21).

(21) a Dabei bewege ich mich nicht [...] mit dem Bauch voran und dem Kopf nach oben, sondern in der durch uraltes Herkommen geheiligten Wirbeltierhaltung mit dem Rücken zum Himmel gewendet und mit dem Kopf nach vorn. (KOL1)

b *Der Rücken zum Himmel gewendet und *der Kopf nach vorn - das ist die typische Körperhaltung der Wirbeltiere, im Unterschied zu * dem Bauch voran und *dem Kopf nach oben.

Die obigen Beispiele veranschaulichen eine weitere, über die SC-Struktur hinausgehende Gemeinsamkeit von MIT-Sätzchen und Absolutkonstruktionen: die charakteristische Pertinenz-/Zugehörigkeitsrelation (im weiteren Sinne), die zwischen einer SC-internen DP als Possessum - dem Bauch usw. in (21 a), der Hand in (19), den Blick in (14D) - und einer Bezugs-DP (meistens dem Subjekt) im Matrixsatz - ich in (21a) und (19), Seff in (14D) - als Possessor besteht (vgl. Zifonun/Hoffmann/Strecker 1997, S. 2224-2226; siehe auch Bech 1955, S. 56 f.). Sie kommt dadurch zustande, dass die SC-interne DP, wenn es sich nicht um ein (Reflexiv-)Pronomen handelt (siehe oben), als Determinativ ein entsprechendes Possessivum oder damit äquivalentes Vorkommen des definiten Artikels enthält. Im typischen Fall bezeichnet die Bezugs-DP ein Animatum und die gebundene SC-interne DP einen Körperteil oder ein anderes, unveräußerliches' Possessum.

\subsection{Ein zweiter Sonderfall: mit/med + AP + N}

Die mit/med-Phrasen, die in den folgenden Beispielen durch Kursivschrift hervorgehoben sind, veranschaulichen einen zweiten von kanonischen Präpositionalphrasen abweichenden Konstruktionstyp, der im Parallelkorpus gleichfalls unter anderem als Übersetzung bzw. Vorlage von Absolutkonstruktionen vorkommt; vgl. (22F) vs. (22N, D).

(22) N Med sin stolthet og standsbevissthet sørget hun oppriktig og med rodkantede oyne da Ingeborg døde. (HW2)

D Mit ihrem Stolz und Standesbewusstsein trauerte sie aufrichtig und mit rotgeweinten Augen, als Ingeborg starb.

F Sa fierté et sa conscience sociale la fit ihr stolz und ihr bewusstsein sozial.FEM sie machte pleurer Ingeborg, les yeux rongis, et avec weinen-über Ingeborg, die augen gerötet, und mit sincérité, quand celle-ci mourut. aufrichtigkeit, als diese starb 

$\mathrm{N}$ Markien
tar imot beskjeden
med bortvendt ansik.t. (NF1)
Marquis.DEF empfängt nachricht.DEF mit abgewandt gesicht
D Mein Herr nahm die Nachricht entgegen.
E He received the news mit abgewandtem Kopf with his eyes averted.
$\mathrm{N}$ Skredderen satt på bordet med iturevet skjorte. (Sæbø et al. i.Dr.) schneider.DEF saß auf tisch.DEF mit zerrissen hemd
D Der Schneider saß mit zerrissenem Hemd auf dem Tisch.

Das Komplement von MIT hat hier die Form einer durch ein vorangestelltes Adjektiv-/Partizipialattribut (AP) erweiterten, scheinbar normalen Nominalphrase - allerdings ohne Artikel oder dergleichen auch in den Fällen, wo ein zählbares Kopfnomen im Singular - ansikt, Gesicht'/Kopf (23N, D) bzw. skejorte/Hemd (24N, D) - vorliegt und ein (expliziter) Artikel folglich zur Bildung einer DP nötig wäre. Das Komplement ist mit anderen Worten keine vollständige DP und deshalb (wenn im Singular) auch nicht in typischen DPFunktionen, d.h. etwa als Subjekt oder Objekt, verwendbar; vgl. (23'a) und (24’b). Und umgekehrt lässt sich das MIT-Komplement ceteris paribus nicht ohne Bedeutungsänderungen durch eine entsprechende definite oder indefinite DP ersetzen: Die Pertinenzrelation, die in (23) und (24) zwischen der MIT-Phrase und dem Satzsubjekt besteht, liegt in $\left(23^{\prime} \mathrm{b}-\mathrm{c}\right)$ und $\left(24^{\prime} \mathrm{b}-\mathrm{c}\right)$ nicht (eindeutig) vor: Anders als in (24) handelt es sich in (24'b-c) nicht (notwendigerweise) um ein/das Hemd, das der Schneider selber anhat; und $\left(23^{\prime} \mathrm{b}-\mathrm{c}\right)$ sind nicht leicht zu interpretieren. ${ }^{16}$

a $*$ Er sah abgewandten Kopf.

b Mein Herr nahm die Nachricht mit einem abgewandten Kopf entgegen.

c Mein Herr nahm die Nachricht mit dem abgewandten Kopf entgegen.

(24') a *Zerrissenes Hemd lag auf dem Stuhl.

b Der Schneider saß auf dem Tisch mit einem zerrissenen Hemd.

c Der Schneider saß auf dem Tisch mit dem zerrissenen Hemd.

Seiner scheinbaren NP-Struktur zum Trotz verhält sich das MIT-Komplement, d.h. [AP N], in Fällen wie (22) bis (24) semantisch offensichtlich weder wie eine DP noch wie eine NP (d.h. ein Prädikat), sondern wie das zweigliedrige (SC-)Komplement in MIT-Sätzchen: $\mathrm{N}$ ist semantisch definit und bezieht sich - wie eine definite bzw. (reflexiv-)possessiv determinierte DP im MIT-Sätzchen - auf ein dem Matrixsubjekt zugeordnetes Possessum, und die AP wird - wie der Prädikatsteil im MIT-Sätzchen - als Prädikat über diese

16 Artikellose Komplemente finden sich - mit dem gleichen semantischen Effekt - auch bei der Präposition OHNE, die allgemein als, lexikalische Negation' von MIT zu analysieren ist; vgl. Der Schneider saß ohne Hemd auf dem Tisch. 
Entität gedeutet. (Dass das [AP N]-Komplement (auch) syntaktisch keine normale DP oder NP ist, sieht man auch daran, dass es sich nicht durch einen Relativsatz erweitern lässt; vgl. *Er verließ ohne Hemd, das er in der Umkleidekabine vergessen hatte, das Geschäft. ${ }^{17}$ ) Mit anderen Worten: MIT-Sätzchen und MIT[AP N] sind als syntaktische (Oberflächen-)Varianten ein und desselben Konstruktionstyps zu bewerten - wie denn auch unter Umständen beide Konstruktionen möglich sind; vgl. (23N') als Alternative zu (23N). Auf die Faktoren, die ihre Verteilung (z.T. sprachspezifisch) regeln mögen, können wir jedoch hier nicht eingehen, und zwar schon deswegen nicht, weil zur MIT[AP N]-Konstruktion, die unseres Wissens überhaupt erst von Sæbø et al. (i.Dr.) in ihrer Eigenart erkannt wird, ${ }^{18}$ noch keine systematischen Untersuchungen vorliegen.

$$
\begin{array}{ll}
\text { N' Markien tar imot beskjeden } \\
\text { marquis empfängt nachricht.DEF }
\end{array} \quad \begin{aligned}
& \text { med ansiktet bortvendt. } \\
& \text { mit gesicht.DEF abgewandt }
\end{aligned}
$$

\subsection{Konstruktionelle Vielfalt der Übersetzungskorrespondenzen}

Absolutkonstruktionen sind nicht die einzigen nicht-analogen Übersetzungen bzw. Vorlagen von MIT-Sätzchen und MIT[AP N]-Konstruktionen, die im OMC registriert werden konnten. Insgesamt ist vielmehr, neben, normalen' MIT-Präpositionalphrasen wie med et nebbete smil, mit einem kessen Lächeln' in (25N), eine ganze Palette mehr oder weniger satzähnlicher Konstruktionen zu verzeichnen, die dementsprechend auch als Übersetzungsbilder voneinander erscheinen:

- Absolutkonstruktionen (in denjenigen Sprachen, die solche besitzen), darunter absolute Akkusative im Deutschen; vgl. (25D), (26D), (31E);

- Partizipialkonstruktionen, darunter sog. free ing-adjuncts (Kortmann 1995) im Englischen; vgl. (25E), (30E);

- Adjektive (bzw. Partizipien) als sog. freie Prädikative, wie breitbeinig in (26D);

- finite Nebensätze, darunter nicht zuletzt (temporale) während-Sätze und ihre Entsprechungen in den anderen Sprachen (31D) oder wobei-Sätze im Deutschen (siehe Abschnitt 3), aber unter Umständen auch Relativsätze (27D);

- mit UND koordinierte Verbalphrasen/Sätze (32D);

- (asyndetisch verknüpfte) selbstständige Sätze; vgl. (29D).

\footnotetext{
17 Wir danken einem anonymen Gutachter für diesen Hinweis.
}

18 Dass die Konstruktion unbeachtet geblieben ist, mag damit zusammenhängen, dass es sie so nicht im Englischen gibt. Hier findet man vielmehr den unbestimmten Artikel, was das withKomplement als eine normale DP erscheinen lässt; vgl. Team captain Gro Hammerseng played with a broken nose (Sæbø et al. i.Dr.) Aus semantischen Gründen ist der indefinite Artikel jedoch in solchen Fällen ,spurious“ (siehe ebd.) 
(25) E The woman waited, facing bim and wearing a perky smile, with her fingers laced together on the counter. (AT1)

D Die Frau wartete, ibm zugewandt, ein kesses Lächeln im Gesicht, die Finger auf der Theke verschränkt.

$\mathrm{N}$ Piken ventet, så på ham med et nebbete smil, mädchen.DEF wartete, sah auf ihn mit einem kessen lächeln, med fingrene foldet på disken.

mit finger.PL.DEF verschränkt auf theke.DEF

(26) N De andre karene satt med skrevende bein og die anderen kerle saßen mit spreizenden bein.PL und overkroppen ruvende over bordet. (HW1) oberkörper.DEF lümmelnd über tisch.DEF

D Die anderen Männer saßen breitbeinig da, den Oberkörper auf den Tisch gelïmmelt.

E The other men sat with their legs sprawled and their chests looming over the table.

(27) N Rett bak henne pustet og strevde veslebroren

$$
\begin{array}{lll}
\text { med kajakken over } & \text { hodet. (MN1) } \\
\text { mit kajak.DEF } & \text { über } & \text { kopf.DEF }
\end{array}
$$

D Direkt hinter ihr keuchte und kämpfte ihr kleiner Bruder, der seinen Kajak auf dem Kopf trug.

$\mathrm{N}$ Jason Coward betraktet den sovende med tvil $i$ Jason Coward betrachtete den schlafenden mit zweifel in gynene.(EFH1) augen.DEF

D Zweifelnd betrachtete Jason Coward den Jungen.

E Jason Coward looked doubtfully at the sleeping boy.

(29) D Mehrere angeschossene Bläßhühner waren ins Schilf gerudert, der Hund sollte sie aufstöbern und apportieren, doch jedesmal, wenn das schwarze Wasser seine Flanken erreichte, sah er winselnd zurück, sein Fell sträubte sich, er verweigerte den Befehl, am Tataren-See, ja ... (SL1)

N [...] begynte den å pistre, snudde seg og så på ham, begann er zu winseln, drehte sich und sah auf ihn, med pelsen strittende,...

mit fell.DEF zu-berge-stehend

(30) E The smoke was very thick and when I made it to the kitchen, coughing and smarting in the eyes, I discovered that the place was on fire. (BO1)

D Der Rauch war sehr dicht, und als ich bustend und mit brennenden Augen in die Küche kam, stellte ich fest, daß sie in Flammen stand. 
(31) N Sol mumler halvhøyt med pekefingeren strittende mot Sol murmelt halblaut mit zeigefinger.DEF zeigend auf avispapiret og naturen rett under den lubne zeitung.DEF und natur.DEF direkt unter dem molligen bakenden. (HW1)

Hintern.DEF

D Sol murmelt halblaut, während sie den gestreckten Zeigefinger gegen das Zeitungspapier und die Natur, direkt unter dem molligen Hintern, drückt.

$\mathrm{E}$ Sol mumbled to herself, her index finger struggling with the piece of newspaper and nature right below her chubby bottom.

$\mathrm{N}$ [...], hun står ensom og med et melankolsk drag $i$ ..., sie steht einsam und mit ART melancholisch zug in ansiktet. (JW1) gesicht.DEF

D Auf den ersten Blick stellt es [das Gemälde] ein Mädchen am Tresen einer Bar dar, das Mädchen ist allein und hat einen melancholischen Gesichtsausdruck.

Diese Vielfalt wirft Fragen auf, die über die Analyse nicht-kanonischer MITKonstruktionen weit hinausgehen und hier nur noch partiell und äußerst knapp kommentiert werden können. ${ }^{19}$

- Was ist den syntaktisch verschiedenen Konstruktionstypen funktional gemeinsam?

- Welche strukturellen und damit zusammenhängenden semantischen Unterschiede bestehen zwischen ihnen?

- Wie kommt die Übersetzungsäquivalenz in Anbetracht solcher Unterschiede (wenn überhaupt) zustande?

- Was regelt die Wahl zwischen verschiedenen Optionen in konkreten Kontexten bzw. beim Übersetzen?

- Inwiefern unterscheiden sich (scheinbar) analoge Konstruktionstypen beispielsweise Absolutkonstruktionen im Deutschen und Englischen über Sprachen hinweg?

Aus funktionaler Sicht haben MIT-Phrasen, Absolutkonstruktionen, Partizipialkonstruktionen und sog. depiktive Adjektive gemeinsam, dass sie der Elaborierung von Ereignisbeschreibungen (im weitesten Sinne, Zustandsbeschreibungen eingeschlossen) dienen (können), sei es, indem sie inhärente Aspekte des durch das Matrixprädikat und dessen Argumente eingeführten Ereignisses präzisieren oder indem sie ein weiteres, grundsätzlich unabhängiges Ereignis, einführen, an dem der einschlägige (Subjekt-)Aktant des Matrixereignisses zugleich beteiligt ist. Im ersten Fall haben wir es in traditioneller Terminologie weitgehend mit Adverbialien des Mittels bzw. der Art

$19 \quad$ Für ausführlichere Diskussionen sei auf Fabricius-Hansen/Haug (Hg.) (i.Dr.) verwiesen. 
und Weise („manner") zu tun, die Wie?-Fragen beantworten können; Beispiele wären die med/with-Sätzchen in $(26 \mathrm{~N}, \mathrm{E})$ sowie med tvil $i$ gynene, mit Zweifel in den Augen' in (28N). Im zweiten Fall werden unsere Konstruktionen je nach Erscheinungsform und syntaktisch/prosodischer Integration gewöhnlich als freie Prädikate oder Depiktiva bzw. als adverbiale Umstandsangaben (,attendant/accompanying circumstances") oder als ,ereignisbezogene Zusätze“ (Zifonun/Hoffmann/Strecker 1997), ,free adjuncts“ (Kortmann 1995) oder dergleichen klassifiziert; Beispiele bieten etwa (30) und (31).

Die traditionellen Termini sind jedoch, wie Himmelmann und SchultzeBerndt (2005) zu Recht bemerken, begrifflich unscharf. Die dort eingeführte, semantisch fundierte Unterscheidung zwischen partizipantenorientierten und ereignisorientierten Adjunkten helfen jedoch auch nicht unbedingt weiter. So bleibt unklar, wie breitbeinig und der absolute Akkusativ in (26D) - die Übersetzungen jeweils des ersten und des zweiten Konjunkts der med-Phrase in $(26 N)$ - zu kategorisieren wären. Einer Klärung bedarf nicht zuletzt das Zusammenspiel zwischen syntaktischer, prosodisch-informationsstruktureller und semantischer Integration solcher Konstruktionen.

MIT-Sätzchen können wie normale Präpositionalphrasen syntaktischprosodisch voll in den Matrixsatz integriert werden, und zwar nicht nur als Adverbialien, sondern im Unterschied zu Absolutkonstruktionen auch als (nachgestellte) Nominalattribute; vgl. (33); bei Absolutkonstruktionen scheint die syntaktisch-prosodische Integrierbarkeit auf Satz- bzw. VP-Ebene hingegen sprachspezifisch zu variieren. Das heißt, der präpositionale Anschluss ermöglicht gemeinhin die syntaktische Integration, die für Absolutkonstruktionen nicht automatisch gegeben ist. So kommt der absolute Akkusativ im Deutschen praktisch nur als ,ereignisbezogener Zusatz“ vor (Gadourek 2006), während Absolutkonstruktionen im Französischen als Adverbialien integriert werden können; vgl. (34). Zur Klärung dieser und verwandter Fragen sind jedoch weitere empirische Untersuchungen nötig.

(33) N $\quad[.$.$] og en småjente med skoleransel på ryggen kom styrtende inn i$ rettslokalet. (BHH1)

D [...], und ein kleines Mädchen mit einem Schulranzen auf dem Rücken kam hereingestürzt.

E $\quad[. .$.$] and a little girl with a schoolbag on her back came rushing into the$ room.

(33’) D * und ein kleines Mädchen einen Schulranzeen auf dem Rücken kam hereingestürzt

E' * and a little girl a schoolbag on her back came rushing into the room

F La nuit, faut-il dormir les fenêtres ouvertes? (Google) die nacht, soll man schlafen die fenster offen.PL

D Soll man nachts mit offenen Fenstern/ mit den Fenstern offen schlafen?

D'*Soll man nachts offene Fenster/ die Fenster offen schlafen? 


\subsection{Konklusion}

Unsere Betrachtungen fingen mit einer Parallelkorpusuntersuchung der Präposition MIT (Abschnitt 4.1) an. Aufgrund syntaktisch variierender Übersetzungskorrespondenzen, die dadurch zutage gefördert wurden, haben sich nun als umfassenderes Sprachen vergleichendes Forschungsfeld die „Elaborierung von Ereignisbeschreibungen“ (im weitesten Sinne) und ihre sprachlichen Ausdrücke abgezeichnet. Für einen systematischen und empirisch fundierten intra- und interlingualen Vergleich einschlägiger Konstruktionen reichen (Parallel-)Korpora mit Wortartenannotierung (wie die Glossa-Version des OMC, siehe Abschnitt 2) jedoch nicht aus. Um die relevanten Konstruktionen gezielt suchen und die Restriktionen, denen sie jeweils unterliegen, bestimmen zu können, sind zusätzliche Informationen syntaktischer, prosodisch-informationsstruktureller und im Idealfall auch diskursfunktionaler Art nötig. ${ }^{20}$

\section{Fazit}

In diesem Beitrag wurde das Oslo Multilingual Corpus vorgestellt, eine mehrsprachige Parallelkorpusressource aus bi- bzw. mehrdirektionalen Teilkorpora, aus u.a. englischen, norwegischen, deutschen und französischen Texten, die seit etwa zwanzig Jahren an der Universität Oslo entwickelt wird (Abschnitt 2). Das OMC eignet sich besonders für kontrastive und sprachvergleichende Untersuchungen, die an konkreten lexikalischen Einheiten wie z.B. Konnektoren, Verben oder Präpositionen festgemacht werden können oder diese als Ausgangspunkt nehmen, was u.a. damit zusammenhängt, dass die Teilkorpora noch nicht durchgängig nach Wortklassenzugehörigkeit annotiert sind und auch avanciertere linguistische Annotationen erst eingeschränkt verfügbar sind. Es wurden zwei OMC-Anwendungsbeispiele präsentiert, eine zielgerichtete korpusbasierte Untersuchung zum deutschen komitativen Konnektor wobei und dessen Übersetzung ins Norwegische bzw. aus dem Norwegischen (Abschnitt 3) und eine explorative Studie, die sich, von der prototypischen komitativen Präposition mit (bzw. med, with, ...) ausgehend, mit satzähnlichen, aber nicht finiten Mitteln zur Elaborierung von Ereignisbeschreibungen befasst (Abschnitt 4). Wir hoffen, in diesem Beitrag gezeigt zu haben, dass ein Parallelkorpus auch ohne komplexe Annotierungen (wie die hier verwendete TCE-Version des OMC) nicht nur für wort-basierte quantitative Untersuchungen verwertet werden, sondern auch im Zuge weniger zielgerichteter, eher qualitativ angelegter Studien als „Augenöffner" für komplexe linguistische Phänomene dienen kann.

20 Eine Mehr-Ebenen-Annotation der erwünschten Art wird zum Beispiel im Rahmen des SFB 632 „Informationsstruktur“ an der Universität Potsdam entwickelt (siehe www.sfb632. uni-potsdam.de). 


\section{Zitierte Korpustexte}

AT1: Tyler, Anne (1985): The Accidental Tourist. New York.

Dt. Übersetzung (1991): Die Reisen des Mr. Leary. Frankfurt a.M.

No. Übersetzung: Roald, Bodil (1986): Tilfeldig turist. Oslo.

BB1: Barth, Boris (1999): Norwegen und der Norden in der deutschen Strategie 1914 bis 1945. In: Simensen, Jarle (1999): Deutschland - Norwegen: Die lange Geschichte. Oslo, S. 165-186.

No. Übersetzung: Rolf Hobsen (1999): Norge og Norden i tysk strategi, 19141945. In: Simensen, Jarle (1999) (Hg.): Tyskland - Norge: Den lange historien. Oslo, S. 144-163.

BHH1: Haff, Bergljot Hobæk (1996): Skammen. Oslo.

Dt. Übersetzung: Haefs, Gabriele (1998): Scham. Hildesheim.

En. Übersetzung: Lyngstad, Sverre (1999): Shame. London.

Fr. Übersetzung: Eydoux, Eric (2000): La Honte. Larbey.

BO1: Okri, Ben (1991): The famished road. London.

Dt. Übersetzung: Wittmann, Uli (1994): Die hungrige Straße. Köln.

CH1: Christoph Hein (1982). Der fremde Freund. Berlin.

No. Übersetzung: Wessel, Elsbeth (1985): Drageblod. Oslo.

EFH1: Hansen, Erik Fosnes (1990): Salme ved reisens slutt. Oslo.

Dt. Übersetzung: Scherzer, Jörg (1995): Choral am Ende der Reise. Köln.

HW1: Herbjørg Wassmo (1981): Huset med den blinde glassveranda. Oslo.

Dt. Übersetzung (1984): Das Haus mit der blinden Glasveranda. München.

HW2: Herbjørg Wassmo (1992). Dinas bok. Oslo.

Dt. Übersetzung: Sack, Ingrid (1992): Das Buch Dina. München.

En. Übersetzung: Christensen, Nadia M. (1996): Dina's Book. Reading.

Fr. Übersetzung: Hinsch, Luce (1994): Le livre de Dina. Paris.

JW1: Wiese, Jan (1991): Kvinnen som kledte seg naken for sin elskede. Oslo.

Dt. Übersetzung: Bruns, Alken (1992): Die Heilige und die Hure. Hamburg.

KF1: Knut Faldbakken (1978): Adams dagbok. Oslo.

Dt. Übersetzung: Stöbling, Ruth (1988): Adams Tagebuch. Rostock.

KOL1: Lorenz, Konrad (1963): Das sogenannte Böse. Wien.

Dt. Übersetzung: Valum, Brynjulf (1968): Den såkalte ondskap. Oslo.

ME1: Ende, Michael (1979): Die unendliche Geschichte. Stuttgart.

No. Übersetzung: Krogstad, Erik (1983): Den uendelige historie. Oslo.

MN1: Newth, Mette (1987): Bortførelsen. Oslo.

Dt. Übersetzung: Haefs, Gabriele (1989): Menschenraub. Aarau.

NF1: Frobenius, Nikolaj (1996): Latours katalog. Oslo.

Dt. Übersetzung: Frauenlob, Günther (1998): Der Anatom. München.

ROS1: Schneider, Robert (1994): Schlafes Bruder. Leipzig.

No. Übersetzung: Dahl, Sverre (1996): Søvnens bror. Oslo. 
SH1: Holmås, Stig (1985): Tordensønnen. Oslo.

Dt. Übersetzung: Schneider, Lothar (1991): Donnersohn. Stuttgart.

SL1: Lie, Sissel (1988): Løvens hjerte. Oslo.

Dt. Übersetzung: Arz, Astrid (1991): Das Herz des Löwen. Hamburg.

\section{Literatur}

Aa, Leiv Inge (2006): Med som innleiande funksjonsord til småsetningar med og utan relativisering. In: Norsk Lingvistisk Tidsskrift 24, S. 193-228.

Bech, Gunnar (1955): Studien über das deutsche verbum infinitum. (= Det Kongelige Danske Videnskabernes Selskab, Historisk-filologiske Meddelelser 35, 2). Kopenhagen.

Blühdorn, Hardarik (i.d.Bd.): Zur Syntax adverbialer Satzverknüpfungen: Deutsch Italienisch - Portugiesisch.

Brandt, Margareta. (1990): Weiterführende Nebensätze. Zu ihrer Syntax, Semantik und Pragmatik. Stockholm. (= Lunder germanistische Forschungen 57).

Breindl, Eva (i.Vorb.): Komitative Konnektoren. In: Breindl, Eva/Volodina, Anna/Waßner, Ulrich: Handbuch der deutschen Konnektoren. Bd. 2: Semantik. Berlin/New York.

Chuquet, Hélène/Chuquet, Jean (2006): De la ,circonstance‘ à la ,cause‘: les prédications en with et leur traduction en français. In: Chuquet, Hélène/Paillard, Michel (Hg.): Causalité et contrastivité: études de corpus. Rennes, S. 189-210.

Dyvik, Helge (1998): A translational basis for semantics. In: Johansson/Oksefjell (Hg.), S. $51-86$.

Ebeling, Jarle (1998): The Translation Corpus Explorer: a browser for parallel texts. In: Johansson/Oksefjell (Hg.), S. 111-112.

Ebeling, Jarle (2000): Presentative constructions in English and Norwegian. A corpusbased contrastive study. (= Acta Humaniora 68). Oslo.

Fabricius-Hansen, Cathrine (1999): Bei dieser Gelegenheit - on this occasion - ved denne anledningen. German bei-a puzzle in a translational perspective. In: Hasselgård, Hilde/ Oksefjell, Signe (Hg.): Out of corpora: studies in honour of Stig Johansson. (= Language and Computers 26). Amsterdam u.a., S. 231-248.

Fabricius-Hansen, Cathrine (2004): Einmalige und ,wiederkehrende` Ereignisse. Über wieder, again, igjen und Verwandtes. In: Lindemann, Beate/Letnes, Ole (Hg.): Diathese, Modalität, Deutsch als Fremdsprache. Festschrift für Oddleif Leirbukt zum 65. Geburtstag. Tübingen, S. 169-180.

Fabricius-Hansen, Cathrine (2005): Elusive connectives. A case study on the explicitness dimension of discourse coherence. In: Linguistics 43, 1, S. 17-48.

Fabricius-Hansen, Cathrine/Haug, Dag T.T. (Hg.) (i.Dr.): Big events, small clauses: the grammar of elaboration. Berlin/New York. 
Gadourek, Thomas M. (2006): Mit satzartigen Konstruktionen unter der Lupe: Über das mit-Sätzchen und dessen Verhältnis zum absoluten Akkusativ. Masterarb., Univ. Oslo.

Giesebrecht, Kjersti Liseth (1999): „Dadurch, dass das eine geschieht, geschieht zugleich das andere“: Eine übersetzungsbezogene Untersuchung zur Funktion der Konjunktion indem im Grenzbereich der modalinstrumentalen, komitativen und temporalen Adverbialsätze. Hovedoppg., Univ. Oslo.

Grabski, Michael/Stede, Manfred (2006): Bei: Intraclausal coherence relations illustrated with a German preposition. In: Discourse Processes 41, 2, S. 195-219.

Hasselgård, Hilde (2007): Thematic structure in translation between English and Norwegian. In: Teubert, Wolfgang/Ramesh, Krishnamurthy (Hg.): Corpus linguistics: critical concepts in linguistics. 6 Bde. London, S. 191-212.

Himmelmann, Nikolaus P./Schultze-Berndt, Eva (2005): Issues in the syntax and semantics of participant-oriented adjuncts: an introduction. In: Himmelmann, Nikolaus P./Schultze-Berndt, Eva (Hg.): Secondary predication and adverbial modification: the typology of depictives. Oxford u.a., S. 1-67.

Hofland, Knut/Johansson, Stig (1998): The Translation Corpus Aligner: a program for automatic alignment of parallel texts. In: Johansson/Oksefjell (Hg.), S. 87-100.

Holler, Anke (2005): Weiterführende Relativsätze. Empirische und theoretische Aspekte. (= Studia Grammatica 60). Berlin.

Jespersen, Otto (1940): A Modern English Grammar: On Historical Principles. Kopenhagen.

Johansson, Stig (1998): On the role of corpora in cross-linguistic research. In: Johansson/Oksefjell (Hg.), S. 3-24.

Johansson, Stig (2007): Seeing through multilingual corpora: on the use of corpora in contrastive studies. (= Studies in Corpus Linguistics 26). Amsterdam.

Johansson, Stig/Oksefjell, Signe (Hg.) (1998): Corpora and cross-linguistic research: theory, method and case studies. (= Language and Computers 24). Amsterdam u.a.

Kortmann, Bernd (1995): Adverbial participial clauses in English. In: Haspelmath, Martin/König, Ekkehard (Hg.): Converbs in cross-linguistic perspective: structure and meaning of adverbial verb forms - adverbial participles, gerunds. (= Empirical Approaches to Language Typology 13). Berlin u.a., S. 189-238.

Løken, Berit (2007): Beyond modals: a corpus-based study of English and Norwegian expressions of possibility. Diss., Univ. Oslo.

McCawley, James (1983): What's with with? In: Language 59, S. 271-287.

Nygaard, Lars et al. (2008): Glossa: a multilingual, multimodal, configurable user interface. In: Proceedings of the Sixth International Language Resources and Evaluation (LREC 2008) in Marrakech, 26th May-1st June 2008, S. 617-622.

Oksefjell, Signe (1999): A description of the English-Norwegian Parallel Corpus: compilation and further developments. In: International Journal of Corpus Linguistics 4, 2, S. 197-219. 
Peyer, Ann (1997): Satzverknüpfung - syntaktische und textpragmatische Aspekte. (= Reihe Germanistische Linguistik 178). Tübingen.

Ramm, Wiebke (2011): Satzgrenzenveränderungen in der Übersetzung: Satzverbindung und lokale Diskursorganisation im Norwegischen und Deutschen. Diss., Univ. Oslo.

Ramm, Wiebke (i.Dr.): German wobei-clauses in translation perspective. In: FabriciusHansen/Haug (Hg.).

Ruwet, Nicolas (1978): Une construction absolue en français. In: Linguisticae Investigationes 11, S. 165-210.

Solstad, Torgrim (2007): Mehrdeutigkeit und Kontexteinfluss: die Spezifikation kausaler Relationen am Beispiel von ,durch'. Diss., Univ. Oslo. Internet: www.ims.unistuttgart.de/ torgrim/publications.shtml.en (Stand: 19.07.2011).

Stolz, Thomas (1997): Some instruments are really good companions - some are not: on syncretism and the typology of instrumentals and comitatives. In: Theoretical Linguistics 23, S. 113-200.

Stolz, Thomas/Stroh, Cornelia/Urdze, Aina (Hg.) (2006): On Comitatives and Related Categories: A Typological Study with Special Focus on the Languages of Europe. Berlin.

Sæbø, Kjell Johan/Haug, Dag T.T./Fabricius-Hansen, Cathrine (i.Dr.): Closed adjuncts; degrees of pertinence. In: Fabricius-Hansen/Haug (Hg.).

Vuillaume, Marcel (1995): Der absolute Akkusativ. In: Eichinger, Ludwig M./Eroms, Hans-Werner (1995): Dependenz und Valenz. (= Beiträge zur germanistischen Sprachwissenschaft 10). Hamburg, S. 397-412.

Zifonun, Gisela/Hoffmann, Ludger/Strecker, Bruno (1997): Grammatik der deutschen Sprache, Bd. 1-3. (= Schriften des Instituts für Deutsche Sprache 7). Berlin u.a. 
